\title{
A reflection on the actual place of osimertinib in the treatment algorithm of EGFR-positive non-small cell lung cancer patients
}

\author{
Alessio Cortellini ${ }^{1,2}$, Corrado Ficorella ${ }^{1,2}$, Roberto Crisci $^{3}$, Duilio Divisi $^{3}$ \\ ${ }^{1}$ Medical Oncology Unit, San Salvatore Hospital, University of L'Aquila, L'Aquila, Italy; ${ }^{2}$ Department of Biotechnological and Applied Clinical \\ Sciences, University of L’Aquila, L'Aquila, Italy; ${ }^{3}$ Thoracic Surgery, University of L'Aquila, “G. Mazzini” Hospital of Teramo, Teramo, Italy \\ Correspondence to: Duilio Divisi, MD. Thoracic Surgery, University of L’Aquila, "G. Mazzini” Hospital of Teramo, Piazza Italia 1, 64100 Teramo, \\ Italy. Email: duilio.divisi@aslteramo.it. \\ Comment on: Eide IJZ, Helland Å, Ekman S, et al. Osimertinib in T790M-positive and -negative patients with EGFR-mutated advanced non-small \\ cell lung cancer (the TREM-study). Lung Cancer 2020;143:27-35.
}

Submitted Apr 22, 2020. Accepted for publication May 20, 2020.

doi: $10.21037 /$ jtd-20-1733

View this article at: http://dx.doi.org/10.21037/jtd-20-1733

\section{Introduction}

We read with interest the results of the TREM study, recently published by Eide et al. (1). The TREM was a multi-center, independent, single arm study, enrolling epidermal growth factor receptor $(E G F R)$ mutant, stage IV, non-small cell lung cancer (NSCLC) patients who progressed to at least one previous treatment line with first/second-generation EGFR-tyrosine kinase inhibitors (EGFR-TKIs), such as gefitinib, erlotinib and afatinib. Every patient received osimertinib (a third-generation EGFR-TKI) at the dose of $80 \mathrm{mg}$ once a day, regardless of the T790M mutational status. The primary end-point was the objective response rate (ORR) in the overall population, even if a formal power calculation was not performed. Being a non-comparative study, no conclusive considerations are allowed; however, the subgroup analyses according to the T790M mutational status raises some interesting questions regarding the evolving therapeutic scenario of EGFRpositive NSCLC patients.

Outcomes reliability was supported by comparison with the AURA trial, which enrolled also T790M negative patients (2). Despite some meaningful differences, such as the dose escalation from $20 \mathrm{mg}$ in the first 31 patients, or patients with performance status (PS) $\geq 2$ not eligible, the AURA trial represents the best comparison for the study of Eide and colleagues. The ORR for the overall population of the two studies was indeed aligned (48\% vs. 51\%). Moreover, in both the AURA and TREM trials patients received at least one prior EGFR-TKI and the percentage of T790M positive patients were similar between ( $28 \%$ vs. $26 \%$ ), as like the percentage of baseline non-L858R point mutations/non-exon 19 deletions (5\% both). On the other hand, comparing the clinical outcomes of the two trials according to the T790M mutational status, some intriguing differences emerge. The T790M positive subgroups displayed similar ORR (60\% vs. 61\%) and progression-free survival (PFS) (10.8 vs. 9.6 months), but the T790M-negative patients of the TREM study (1) showed a slightly higher ORR (28\% vs. $21 \%)$ and a prolonged PFS (5.1 vs. 2.8 months). According to the AURA 3 study results, osimertinib should be considered the standard second line treatment only for NSCLC patients who develop a T790M-driven disease progression to first/second-generation EGFR-TKIs (3), whilst the finding of a certain clinical benefit with second line osimertinib in T790M negative patients, might again raise some questions. From this point of view, the TREM study results might suggest that all the patients who received front line first/second-generation EGFR-TKI (including those who develop T790M negative disease progression) could benefit from second line osimertinib, reinforcing the theory that all the EGFR-positive NSCLC patients should be treated with an EGFR-TKIs sequencing across treatment lines. Nevertheless, the recently updated 
Table 1 summary of the rate of patients who received post-progression systemic treatments in clinical trials with EGFR-TKIs

\begin{tabular}{llr}
\hline Study & EGFR-TKI & Post-progression treatment*, \% \\
\hline IPASS, Fukuoka et al., J Clin Oncol 2011 & Gefitinib & 76 \\
NEJ002, Inoue et al., Ann Oncol 2013 & Gefitinib & 72 \\
WJTOG 3405, Yoshioka et al., Ann Oncol 2019 & Gefitinib & 86 \\
EURTAC, Rosell et al., Lancet Oncol 2012 & Erlotinib & 68 \\
OPTIMAL, Zhou et al., Ann Oncol 2015 & Erlotinib & 63 \\
LUX-LUNG 3, Yang et al., Lancet Oncol 2015 & Afatinib & 71 \\
LUX-LUNG 6, Yang et al., Lancet Oncol 2015 & Afatinib & 57 \\
LUX-LUNG 7, Paz-Ares et al., Ann Oncol 2017 & Afatinib & 73 \\
FLAURA, Ramalingam et al., N Engl J Med 2020 & Gefitinib & 77 \\
\end{tabular}

*, After discontinuation of study treatment, excludes patients remaining on assigned treatment at data cut-off, pre-progression treatment and death. EGFR-TKI, epidermal growth factor receptor-tyrosine kinase inhibitor.

FLAURA study confirmed that first line osimertinib lead to a significantly prolonged overall survival (OS) compared to first-generation EGFR-TKIs in NSCLC with common sensitizing mutations (4), refuting, at least partially, the EGFR-TKIs sequencing therapeutic approach.

\section{The EGFR-TKIs sequencing approach}

EGFR-TKIs sequencing is a treatment strategy which provides a sequential use of first/second-generation EGFRTKI as first line treatment, followed by osimertinib as second line in case of T790M-driven disease progression. This strategy has of course some positive aspects. A recent real-world analysis of $576 \mathrm{~T} 790 \mathrm{M}$ positive patients who received first/second-generation TKIs followed by osimertinib, reported a 36-month OS rate of 51\% (5). Careful interest was addressed to the sequence afatinib/ osimertinib: a subgroup analysis of an observational prospective registry, although with an extremely small sample size (29 patients), reported a 24 -month OS rate of $89.3 \%$ for T790M positive patients who received both drugs (6). Similarly, another retrospective real-life study highlighted a median OS of 41.3 months for the overall study population, and of 45.7 months for patients with exon 19 deletion (7). The greatest flaw of the sequential approach is due to not every patients are used to developing T790M driven disease progression, and therefore can benefit from second line osimertinib. Intriguingly, the quite good efficacy reported by the TREM study for T790M negative patients (ORR of $28 \%$ and the PFS of 5.1 months), could make us believe this limitation overcome, supporting the sequencing approach for all. Ideal sequential approach should be offered to each patients; unfortunately, not every patients developed T790M driven disease progression, and therefore cannot benefit from second line osimertinib. However, to proper evaluate our considerations, several aspects must be taken into account. First, we analyzed the rate of patients who usually reach a second line treatment. Disease progression of metastatic NSCLC could be lifethreating, and a not negligible percentage of patients dies at each treatment line. Table 1 summarized the rate of patients who received post-progression systemic treatments in clinical trials with EGFR-TKI (range: 60-86\%). Also, the T790M test availability might be considered a limit to overcome. A recent analysis (8) reported that: (I) $23 \%$ of patients receiving first/second-generation EGFR-TKIs did not received a second line treatment; (II) $25 \%$ of the progressors were not tested for T790M mutation; (III) $41 \%$ of the progressors finally received second line osimertinib. Similarly, Shah et al. (9) reported that $21 \%$ of the progressors to first/second-generation EGFR-TKIs did not received a second line treatment, $26 \%$ of the progressors were not tested for T790M mutation and only $28.2 \%$ of the progressors received second line osimertinib. Therefore, the T790M detection rate at disease progression should always be taken into account. Eide et al. (1) showed $60 \%$ of T790M 
patients which was higher than what reported in some reallife studies $(10,11)$, but aligned to others (12). The updated FLAURA trial (4) experienced a median OS of 38.6 months for the experimental arm by first line osimertinib and a median follow-up of 39 months, which seems shorter than the OS (since the starting of the first line) reported for T790M positive patients receiving sequential first/ second-generation TKIs and osimertinib. Nevertheless, we must to consider some selection bias. Patients developing T790M driven disease progression have a better prognosis, regardless of the treatment with second line osimertinib. A meta-analysis on the impact of acquired EGFR T790M mutation, which included studies where no patients had ever received third-generation EGFR-TKIs, displayed a significantly longer post-PFS, OS and PFS since the first line in T790M positive patients (13). Oxnard et al. (14), in a prospective re-biopsy protocol performed many years before the introduction into clinical practice of thirdgeneration EGFR-TKIs, noticed that T790M positive $v s$. negative patients had a longer post-PFS and a longer time to new metastases. The TREM study (1) revealed a higher T790M detection rate for patients with previous exon 19 deletion (53\%), compared to patients with exon 21-point mutation (26\%), as already reported (15). Moreover, Eide et al. (1) showed, among the T790M negative patients, a prolonged PFS and OS if there were no central nervous system (CNS) metastases compared to CNS involvement. These results assume that a sequencing approach could be considered for patients with baseline exon 19 deletion and without CNS metastases. TREM study (1) enrolled also patients with uncommon mutations, and to date, the only category for whom EGFR-TKIs sequencing should to be considered upfront, is represented by patients with uncommon EGFR mutations (excluding exon 20 mutation). Indeed, the FLAURA study (4) enrolled only patients with common EGFR mutations, and despite some intriguing results have already reported in a phase II study (16), there are not reliable data for such indication yet. Instead, quite good results with afatinib have highlighted across the LuxLung trials (17).

\section{The evolving scenario of EGFR positive NSCLC}

Recently, dacomitinib (second-generation EGFR-TKI) have considered in the first line scenario. Despite some major limitations such as the hierarchical order of the endpoints, the failure to enroll patients with CNS metastases and a not negligible toxicity profile, the phase III ARCHER 1050 study (18) showed an improvement of PFS and OS for first line dacomitinib compared to gefitinib. Even if the T790M detection rate among the progressors to dacomitinib is still not available, only $9.7 \%$ of the patients in the experimental arm received a third-generation TKI as second line treatment at the data cut-off. Combination of firstgeneration EGFR-TKI with anti-angiogenic monoclonal antibodies has proven effective. The phase III studies in treatment of EGFR positive NSCLC patients, comparing erlotinib alone with either erlotinib plus bevacizumab (19) or erlotinib plus ramucirumab (20), have both displayed interesting results in terms of efficacy. However, the same principles and flaws of the sequential approach already discussed, may be applied to this setting. In fact, the T790M detection rate reported among the progressors of the experimental arm of the RELAY study was 43\% (19). Even the combination of gefitinib with platinum-based chemotherapy showed OS significant benefit over gefitinib alone (21), and might be taken into consideration in those countries where first-line osimertinib is not reimbursed. Currently, a phase III study of upfront combination of osimertinib with platinum-based chemotherapy is underway (22). While in the clinical practice of western countries the osimertinib indication is moving forward to the first-line setting, it might think that the avoidance of sequential approach could results in a lack of therapeutic options at disease progression. In our opinion, an early monitoring during treatment with quantitative and qualitative liquid biopsy techniques should be carried out when available, in order to early detect progressors whit actionable biomarkers (23). In case of oligoprogression, when it occurs without actionable biomarkers emergence, osimertinib has already proved its feasibility eventually combined with local ablative treatments (24). The combination of carboplatin/paclitaxel with both bevacizumab and atezolizumab (an anti-programmed death-ligand 1 checkpoint inhibitor) (ABCP) is another therapeutic option to disease progression. The IMpower 150 study (25), enrolling also pre-treated oncogene addicted patients excluded from the primary analysis, revealed impressive efficacy outcomes for the subgroup of EGFR mutation-positive patients. However, the applicability of the ABCP combination in the setting of disease progression during first line osimertinib, is heavily flawed by the previous treatment with first/second-generation EGFRTKI in the IMpower 150 experience (25). Moreover, the T790M status was not available and the sample size was also too low in order to make definitive considerations. 


\section{Conclusions}

In order to make a proper choice among first line treatment options for EGFR positive NSCLC patients, the major points to take into consideration are:

- The rate of patients receiving a second line treatment in both clinical trial and real-life settings;

- The T790M detection rate and the testing availability;

- The T790M-related positive selection bias (when comparing first line osimertinib OS with EGFRTKI sequencing cumulative OS);

- Resources availability and local reimbursement policies.

Despite the interesting results reported by Eide et al. (1) for the second line osimertinib regardless of the T790M mutational status, in our opinion each metastatic EGFR positive NSCLC patients with common mutation (L858R and exon 19 deletions) should receive upfront osimertinib when local regulatory policies allows it.

\section{Acknowledgments}

Funding: None.

\section{Footnote}

Provenance and Peer Review: This article was commissioned by the editorial office, Fournal of Thoracic Disease. The article did not undergo external peer review.

Conflicts of Interest: All authors have completed the ICMJE uniform disclosure form (available at http://dx.doi. org/10.21037/jtd-20-1733). AC received speaker fees and grant consultancies by AstraZeneca, MSD, BMS, Roche, Novartis and Astellas. The other authors have no other conflicts of interest to declare.

Ethical Statement: The authors are accountable for all aspects of the work in ensuring that questions related to the accuracy or integrity of any part of the work are appropriately investigated and resolved.

Open Access Statement: This is an Open Access article distributed in accordance with the Creative Commons Attribution-NonCommercial-NoDerivs 4.0 International License (CC BY-NC-ND 4.0), which permits the noncommercial replication and distribution of the article with the strict proviso that no changes or edits are made and the original work is properly cited (including links to both the formal publication through the relevant DOI and the license). See: https://creativecommons.org/licenses/by-nc-nd/4.0/.

\section{References}

1. Eide IJZ, Helland Å, Ekman S, et al. Osimertinib in T790M-positive and -negative patients with EGFRmutated advanced non-small cell lung cancer (the TREMstudy). Lung Cancer 2020;143:27-35.

2. Jänne PA, Yang JC, Kim DW, et al. AZD9291 in EGFR inhibitor-resistant non-small-cell lung cancer. $\mathrm{N}$ Engl J Med 2015;372:1689-99.

3. Mok TS, Wu YL, Ahn MJ, et al. Osimertinib or platinumpemetrexed in EGFR T790M-positive lung cancer. N Engl J Med 2017;376:629-40.

4. Ramalingam SS, Vansteenkiste J, Planchard D, et al. Overall survival with osimertinib in untreated, EGFR-mutated advanced NSCLC. N Engl J Med 2020;382:41-50.

5. Girard N, Bouee S, Moro-Sibilot D, et al. Effectiveness of sequencing TKis in patients with EGFR mutation-positive non-small-cell lung cancer (NSCLC): a French national medico administrative claim database analysis. Ann Oncol 2019;30:v627-8.

6. Brückl W, Reck M, Laack E, et al. Sequential treatment with Afatinib followed by 3rd generation EGFR-Tki Subgroup analysis of the GIDEO trial: a prospective noninterventional study (NIS) in EGFR mutated NSCLC patients in Germany. Ann Oncol 2019;30:v627.

7. Hochmair MJ, Morabito A, Hao D, et al. Sequential afatinib and osimertinib in patients with EGFR mutation-positive non-small-cell lung cancer: updated analysis of the observational GioTag study. Future Oncol 2019;15:2905-14.

8. Christopoulos P, Volckmar A, Bozorgmehr F, et al. Realworld implementation of sequential targeted therapies for EGFR-mutated NSCLC. Ann Oncol 2019;30:v631-2.

9. Shah R, Girard N, Nagar SP, et al. Real-world (RW) treatment patterns and outcomes for second-line $(2 \mathrm{~L})$ therapy and beyond in patients (PTS) with epidermal growth factor receptor-mutated (EGFRM) advanced NSCLC receiving a first-line (1L) first- or seconfgeneration (1G/2G) EGFR tyrosine kinase inhibitor (TKI). Ann Oncol 2019;30:v624-5.

10. Hong MH, Kim HR, Ahn BC, et al. Real-world analysis of the efficacy of rebiopsy and EGFR mutation test of tissue and plasma samples in drug-resistant non-small cell lung cancer. Yonsei Med J 2019;60:525-34. 
11. Seto T, Nogami N, Yamamoto N, et al. Real-world EGFR T790M testing in advanced non-small-cell lung cancer: a prospective observational study in Japan. Oncol Ther 2018;6:203-15.

12. Hochmair MJ, Buder A, Schwab S, et al. Liquid-biopsybased identification of EGFR T790M mutation-mediated resistance to afatinib treatment in patients with advanced EGFR mutation-positive NSCLC, and subsequent response to osimertinib. Target Oncol 2019;14:75-83.

13. Liu Y, Sun L, Xiong ZC, et al. Meta-analysis of the impact of de novo and acquired EGFR T790M mutations on the prognosis of patients with non-small cell lung cancer receiving EGFR-TKIs. Onco Targets Ther 2017;10:2267-79.

14. Oxnard GR, Arcila ME, Sima CS, et al. Acquired resistance to EGFR tyrosine kinase inhibitors in EGFRmutant lung cancer: distinct natural history of patients with tumors harboring the T790M mutation. Clin Cancer Res 2011;17:1616-22.

15. Liang H, Pan Z, Wang W, et al. The alteration of T790M between 19 del and L858R in NSCLC in the course of EGFR-TKIs therapy: a literature-based pooled analysis. J Thorac Dis 2018;10:2311-20.

16. Cho JH, Lim SH, An HJ, et al. Osimertinib for patients with non-small-cell lung cancer harboring uncommon EGFR mutations: a multicenter, open-label, phase II trial (KCSG-LU15-09). J Clin Oncol 2020;38:488-95.

17. Yang JC, Sequist LV, Geater SL, et al. Clinical activity of afatinib in patients with advanced non-small-cell lung cancer harbouring uncommon EGFR mutations: a combined post-hoc analysis of LUX-Lung 2, LUX-Lung 3, and LUX-Lung 6. Lancet Oncol 2015;16:830-8.

18. Mok TS, Cheng Y, Zhou X, et al. Improvement in overall survival in a randomized study that compared dacomitinib with gefitinib in patients with advanced non-small-cell

Cite this article as: Cortellini A, Ficorella C, Crisci R, Divisi D. A reflection on the actual place of osimertinib in the treatment algorithm of EGFR-positive non-small cell lung cancer patients. J Thorac Dis 2020;12(10):6107-6111. doi: 10.21037/ jtd-20-1733 lung cancer and EGFR-activating mutations. J Clin Oncol 2018;36:2244-50.

19. Zhou Q, Cheng Y, Liu Y, et al. CTONG 1509: Phase III study of bevacizumab with or without erlotinib in untreated Chinese patients with advanced EGFR-mutated NSCLC. Ann Oncol 2019;30:v603.

20. Nakagawa K, Garon EB, Seto T, et al. Ramucirumab plus erlotinib in patients with untreated, EGFRmutated, advanced non-small-cell lung cancer (RELAY): a randomised, double-blind, placebo-controlled, phase 3 trial. Lancet Oncol 2019;20:1655-69.

21. Zhang W, Xu J, Lou Y, et al. Chemotherapy plus EGFRTKI as first-line treatment provides better survival for EGFR mutation NSCLC patients: update data for NCT02148380. J Thorac Oncol 2019;14:S236.

22. AZD9291 versus gefitinib or erlotinib in patients with locally advanced or metastatic non-small cell lung cancer (FLAURA). ClinicalTrials.gov Identifier: NCT02296125. Available online: https://clinicaltrials.gov/ct2/show/ NCT02296125

23. Buttitta F, Felicioni L, Lorito AD, et al. Early prediction of resistance to tyrosine kinase inhibitors by plasma monitoring of EGFR mutations in NSCLC: a new algorithm for patient selection and personalized treatment. Oncotarget 2020;11:982-91.

24. Cortellini A, Leonetti A, Catino A, et al. Osimertinib beyond disease progression in T790M EGFR-positive NSCLC patients: a multicenter study of clinicians' attitudes. Clin Transl Oncol 2020;22:844-51.

25. Reck M, Mok TSK, Nishio M, et al. Atezolizumab plus bevacizumab and chemotherapy in non-small-cell lung cancer (IMpower150): key subgroup analyses of patients with EGFR mutations or baseline liver metastases in a randomised, open-label phase 3 trial. Lancet Respir Med 2019;7:387-401. 